\title{
Tuberculous Cerebellar Abscess
}

\author{
Abayomi G. Osbinowo, MD, B. Wayne Blount, MD, MPH, and Lawrence L. Golusinski, $M D$
}

Background: Tuberculosis is an important illness with an increasing occurrence. Although considered primarily a pulmonary disease, tuberculosis can affect any organ system. Central nervous system involvement is potentially devastating and occurs with escalating frequency in both immunocompetent and immunologically incompetent populations.

Methods: This case report involved a patient admitted to the authors' inpatient service. Data were obtained from the patient's medical record. MEDLINE and Index Medicus literature searches were conducted for the years 1977 to the present, with cross-references for earlier articles.

Results and Conclusions: A 36-year-old previously healthy, immunocompetent woman with a diagnosis of extrapulmonary tuberculosis and no determined primary focus was found to have a cerebellar abscess. Treatment included surgical excision of the abscess combined with 6 months of antituberculosis therapy. Diagnostic tools included the tuberculosis skin test, smears and culture of specimens, computed tomographic scans, and rapid assays based on nucleic acid amplification, ie, polymerase chain reaction. The polymerase chain reaction has great potential for rapid diagnosis of Mycobacterium tuberculosis, particularly when there might be few bacilli, as in pleural, peritoneal, or cerebrospinal fluid. Appropriate therapy for tuberculous cerebellar abscess includes standard antituberculosis medications for 6 to 9 months and surgical excision of the abscess. (J Am Board Fam Pract 1998;11:459-64.)

From 1985 through 1993, the incidence of tuberculosis in the United States increased by 14 percent, but decreased slightly in 1994. Considered primarily a pulmonary disease, tuberculosis can also infect almost any body system through lymphohematogenous spread, ${ }^{1}$ and extrapulmonary tuberculosis now constitutes 18.5 percent of all cases compared with 8 percent of all cases 20 years ago. ${ }^{1}$ The usual signs and symptoms of extrapulmonary tuberculosis are nonspecific, both challenging and delaying diagnosis. ${ }^{1}$

Although extrapulmonary tuberculosis is more often found in immunocompromised persons, otherwise healthy patients can also have it. Tuberculosis has been found in the lymphatic system, pleura, genitourinary system, bones and joints, mastoid, gastrointestinal system, peritoneum, skin, heart, eye, thyroid, tongue, and central nervous system. ${ }^{1}$ In the central nervous system, tuberculosis is usually a meningeal infection or a tuberculoma; rarely is it an abscess, and even more rarely is it a cerebellar abscess. ${ }^{2}$ Diagnosis is fre-

Submitted, revised, 24 November 1997.

From the Department of Family and Preventive Medicine (AGO, BWB, LLG), Emory University School of Medicine, Atlanta. Address reprint requests to Abayomi G. Oshinowo, MD, 478 Peachtree St, NE, Suite 818-A, Atlanta, GA 30308. quently quite delayed in central nervous system involvement.

We encountered a young, healthy woman with cerebellar abscess whose initial complaint was a chronic headache. Chronic headache is considered the seventh most common complaint recorded for ambulatory care encounters in the United States. ${ }^{3}$ Her case underscores that common symptoms can have uncommon causes. In this era of resurgent tuberculosis, we must remain alert for the uncommon, subtle manifestations of this disease, as inclusion of tuberculosis in the differential diagnosis of common complaints requires a high index of suspicion. Fortunately, timely initiation of therapy can lead to reduced morbidity and mortality.

\section{Methods}

We describe a case report that involved a patient admitted to the authors' inpatient service. Data were obtained from the patient's medical record. MEDLINE and Index Medicus literature searches were conducted using the key words "tuberculosis," "brain abscess," "extrapulmonary tuberculosis." and "polymerase chain reaction," for the years 1977 through 1997, with cross-references for earlier articles. 


\section{Case Report}

A 36-year-old woman was admitted to the family medicine inpatient service with a 6-week history of persistent left-sided headaches. Onset of headaches occurred 4 days after extraction of a carious right second molar, and the pain was initially localized to the right frontal region. She described the headache as the worst in her life and characterized the headache as squeezing, throbbing, sharp, and hammerlike. She denied any association of facial swelling or abscess formation with her toothache. After the molar extraction, penicillin was prescribed, but she stopped taking it after four doses because of the onset of the rightsided headache. She denied any associated nausea, vomiting, visual blurring, or fever and any history of headaches or migraines. She further stated that the headaches were not relieved by either regularstrength acetaminophen or acetaminophen in combination with hydrocodone.

Before her admission she had made four trips to two emergency departments. Her headaches had been diagnosed and managed as sinusitis on the first visit, as cluster headache on the second visit, and as temporomandibular joint syndrome on the third visit. On her fourth visit, she was referred to an oral surgeon, who diagnosed muscle spasms and injected her jaw. After the injection her right-sided headache was reduced to a feeling of pressure, but she had an onset of a similar, leftsided headache. Findings during a review of systems were noncontributory and in particular were negative for chronic cough, weight loss, night sweats, fever, or other constitutional symptoms.

Her medical history was notable for hypothyroidism diagnosed 3 years earlier. Her medications included depo-medroxyprogesterone (for contraception), flurbiprofen, hydrocodone, naproxen, and L-thyroxine. She worked as a hair dresser and lived with her 2 children and fiancé. She denied using alcohol, tobacco, or illicit drugs, and her family history was unremarkable. A careful history obtained later revealed possible exposure to tuberculosis. She stated that 3 months before admission, one of her co-workers had been diagnosed with tuberculosis (based on a positive tuberculin skin test and chest radiograph) and was undergoing treatment with a three-drug antituberculosis drug regimen. She further stated that she (and all her other co-workers) had received tuberculin skin testing and had tested negative.
At admission the patient appeared to be acutely ill, alert, and oriented. Her blood pressure was $110 / 70 \mathrm{mmHg}$ with her right arm supine, pulse $78 / \mathrm{min}$ and regular, respiratory rate $16 / \mathrm{min}$, and temperature $37.3^{\circ} \mathrm{C}$. Findings during an examination of the respiratory, cardiovascular, and gastrointestinal systems were within normal limits. Pertinent findings, however, were found during the central nervous system and musculoskeletal system examination. The patient had decreased hearing on the right, slurred speech, right-sided facial droop, numbness in the distribution of the right fifth cranial nerve, a positive Brudzinski sign, submandibular and anterior cervical lymphadenopathy, hyperreflexia, bilateral clonus, and mild weakness of her right hand grip.

Laboratory tests showed a normal complete blood cell count and normal electrolyte levels. Urinalysis results included a protein level of 30 $\mathrm{mg} / \mathrm{dL}$ with 5 to 10 white blood cells and 10 to 15 red blood cells per high-power field. A lumbar puncture had an opening pressure of $32 \mathrm{~cm}$; and examination of the spinal fluid showed a leukocyte count of $469 / \mu \mathrm{L}$ with 14 percent segmented neutrophils, 85 percent lymphocytes, and 1 percent histiocytes; erythrocyte count of $84 / \mu \mathrm{L}$; glucose $12 \mathrm{mg} / \mathrm{dL}$; and protein $165 \mathrm{mg} / \mathrm{dL}$. Tests for rapid plasma reagin, human immunodeficiency virus antibody, cryptococcal antigen, toxoplasmosis antibody, and purified protein derivative all produced negative results. At admission a roentgenogram of the chest showed no abnormalities, and a noncontrast head computed tomogram (CT) showed mild prominence of the ventricular system.

Based on the history of tooth extraction, we prescribed a combination of ceftriaxone, metronidazole, and ampicillin for the most common causes of pyogenic brain abscess: anaerobes, gramnegative rods and gram-positive cocci. Ampicillin was added for Listeria monocytogenes.

When the patient did not improve, she had a magnetic resonance imaging scan (MRI) with gadolinium on hospital day 3 , which showed abnormal signal and enhancement within the right brain stem, pons, midbrain, cerebral peduncle, and middle cerebellar peduncle, with extension into the right internal auditory canal, where there was an area of necrosis and rim enhancement (Figure 1). With the advice of an infectious disease specialist, we decided to continue antibiotics and 


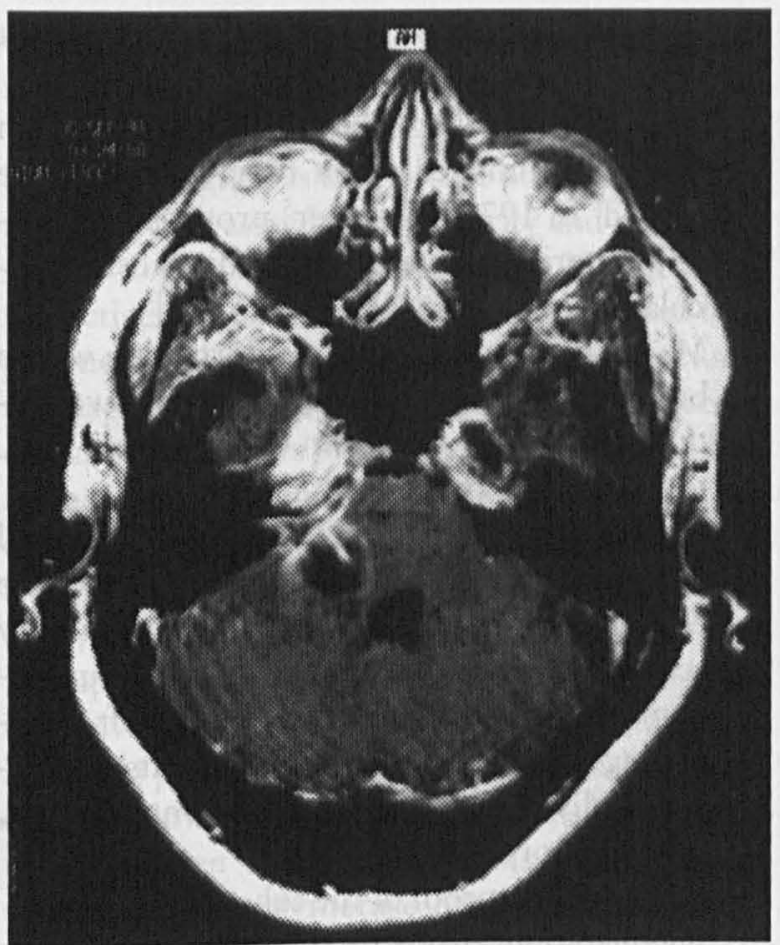

Figure 1. T1-weighted axial images using gadolinium show multiple ring-enhancing lesions in right middle cerebellar peduncle and pons. Minimal mass effect on the fourth ventricle appears with contrast enhancement in the region of the internal auditory canal.

observe her clinical course. Her symptoms still did not improve, and on hospital day 8 , isoniazid (15 $\mathrm{mg} / \mathrm{kg} / \mathrm{d})$, rifampin (10 mg/kg/d), ethambutol (25 $\mathrm{mg} / \mathrm{kg} / \mathrm{d})$ and pyrazinamide $(30 \mathrm{mg} / \mathrm{kg} / \mathrm{d})$ were added empirically for possible tuberculosis. A second MRI on hospital day 9 showed interval progression of the enhancing lesion with increased vasogenic edema and mass effect on the fourth ventricle (Figure 2).

On hospital day 10, ultrasonic probing through a retrosternomastoid dissection showed two distinct abscess cavities, which were drained. Gram stain of the aspirate showed many polymorphonuclear leukocytes but no organisms. Macroscopic examination of the abscess wall showed multiple soft and hemorrhagic friable tissue fragments. Although an acid-fast stain showed no acid-fast bacilli, clinical suspicion was high that this abscess was tuberculous, and a polymerase chain reaction assay for Mycobacterium tuberculosis was performed on the aspirate as previously described. ${ }^{4}$ The assay specifically targets IS 6110 , an insertion enzyme repeated many times in the chromosome of $M$ tuberculosis. The aspirate

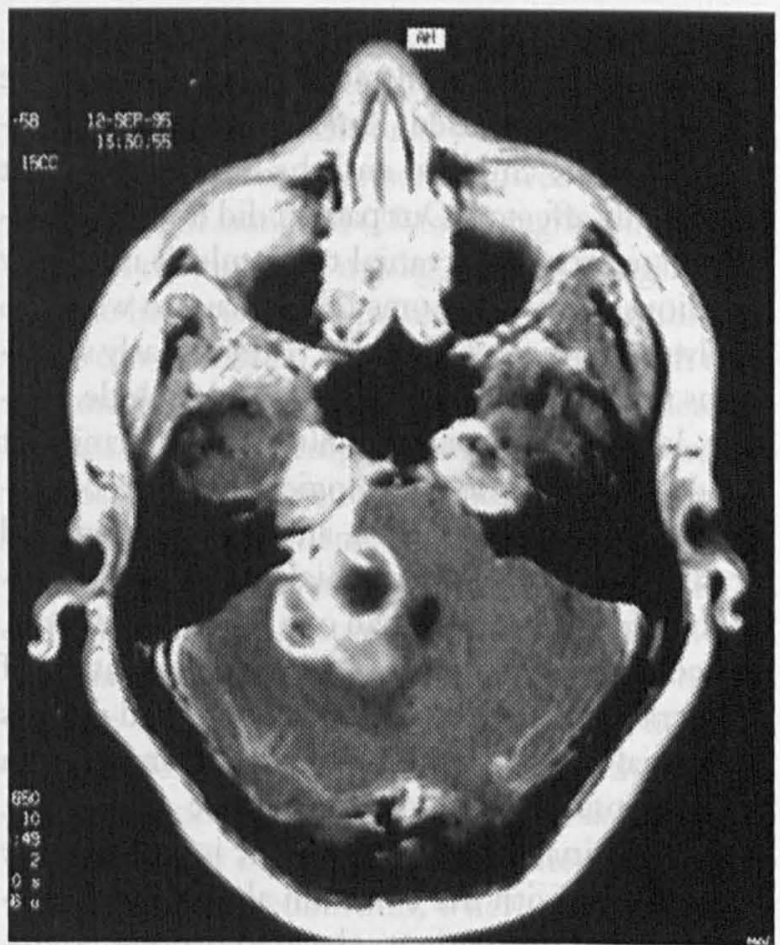

Figure 2. T1-weighted axial image using gadolinium shows progression of the lesion with increased vasogenic edema and mass effect on the fourth ventricle.

was positive only after Southern blot hybridization with a specific oligonucleotide probe.

The patient was given a 3 -week tapering course of dexamethasone and told to continue her four-drug antituberculosis regimen for a total of 2 months. She was discharged home on day 23 with orders for occupational and physical therapy. After she completed 2 months of the four-drug therapy, her regimen was changed to a twice weekly, directly observed treatment of isoniazid $(900 \mathrm{mg})$ and rifampin $(600 \mathrm{mg})$ for an additional 4 months.

One year after discharge the patient has regained function of cranial nerve VII. She still has deficits in the sensory branch of the right fifth cranial nerve and continues to have persistent dryness and recurrent infection of the right eye, which required tarsorrhaphy of the right eyelid. In addition, she continues to have diplopia and visual blurring secondary to a nonprogressive motor deficit of the third right cranial nerve. Today she walks with the assistance of a cane and has right upper extremity weakness and mild deficits in fine motor activities of the right hand. 


\section{Discussion}

Usually patients with intracranial tuberculosis are immunocompromised. Among immunocompetent patients, children and the elderly are most commonly affected. Our patient did not fit into either category. Intracranial tuberculosis is usually insidious, with symptoms taking days to weeks to evolve, as in the case with our patient. Early symptoms are generally nonspecific and include malaise, low-grade fever, irritability, and intermittent headaches. Specific neurologic symptoms occurring later include stiff neck, altered cognition, and cranial nerve palsies. Cranial nerve palsies, although found in fewer than one fourth of patients, are considered the most specific manifestation of intracranial tuberculosis. Our patient had deficits in cranial nerves III, V, VII, and VIII in addition to the constellation of nonspecific symptoms. Cerebrospinal fluid examination is also usually nonspecific, with the common abnormalities exemplified in our patient: elevated protein count, elevated white blood cell count (predominantly a lymphocytosis), and decreased glucose levels.

Although CT and MRI have increased the detection rate of intracranial abscesses, neither these studies, clinical symptoms, nor findings at surgery easily differentiate between tuberculous and pyogenic abscesses. Findings on C'T scans are assumed to be characteristic of tuberculous abscesses if they show a thick-walled lesion with surrounding edema, and pyogenic, if abscess walls are relatively thin. ${ }^{2}$ Our patient's lesion failed to appear on a CT scan but was readily apparent on a MRI scan using gadolinium. Currently there are no published data supporting the routine use of contrast-enhanced MRI when tuberculosis of the central nervous system is suspected. Nevertheless, MRI is better than CT as a method for imaging posterior fossa lesions.

Tuberculous abscesses are more often supratentorial ${ }^{5}$ and are believed to result from localized extension of infection from the sinuses, mastoids, or bone. The primary focus of infection in this case remains in doubt. The patient had both an extraction of a carious tooth and had sinusitis. The brain stem is rarely the site of a focal tuberculous lesion; when it occurs, it is more often a brain stem tuberculoma, and only rarely is a true abscess described. The abscess in our patient was in the area of the right brain stem involving the pons, midbrain, cerebral peduncle, and middle cerebel- lar peduncle, a rare location for the development of a tubercular abscess.

The definitive diagnosis of a tubercular brain abscess is established when tubercle bacilli are identified. In 1978 Whitener ${ }^{5}$ proposed the following criteria for establishing a diagnosis of tubercular brain abscess:

1. Macroscopic evidence of pus formation within brain parenchyma. (Our patient had two distinct abscess cavities, from one of which $3 \mathrm{~mL}$ to $4 \mathrm{~mL}$ of pus were aspirated.)

2. Histologic confirmation that the abscess wall was composed of vascular granulation tissue containing acute and chronic inflammatory cells. (Macroscopic examination of our patient's abscess wall showed multiple soft, hemorrhagic and friable tissue fragments; microscopically there were acute, but no chronic, inflammatory cells.)

3. Bacteriologic proof of the tuberculous origin.

Bacteriologic proof of tuberculous origin is usually difficult, implying a positive finding of acid-fast bacilli on staining and $M$ tuberculosis on culture. The absence of these findings, as in this case, however, does not necessarily imply the absence of a tuberculous brain abscess. Dastur and Desai $^{6}$ recorded a history of tuberculosis infection in only 47 percent of 107 cases, and Arseni $^{7}$ recorded the same in only 52 percent of 101 cases of tuberculous brain abscesses, confirmed retrospectively, on the basis of response to antituberculosis drug therapy.

When tested, our patient was anergic to both the tuberculin skin test and the control panel. Tuberculin skin tests, however, although well established as a means of screening for persons infected with $M$ tuberculosis, now give more than the usual false-negative responses because of the increased occurrence of cutaneous anergy secondary to acquired immunodeficiency syndrome and its associated suppressed immunologic response. Gram stain of the abscess revealed numerous polymorphonuclear leukocytes with no organisms, and staining was negative for acid-fast bacilli. Notably, the patient had begun antimicrobial and antituberculosis therapy prior to culturing. In addition, Gram stain and culture of cerebrospinal fluid for acid-fast bacilli were negative. Direct examination of cerebrospinal fluid for acid-fast bacilli is disappointing, however, with a reported 10 to 40 percent detection rate, which can increase to 87 per- 
cent with three separate specimens of cerebrospinal fluid. ${ }^{8}$

The only evidence that this abscess was of tuberculous origin was the positive polymerase chain reaction. This assay has a specificity and sensitivity similar to culture, 92.1 percent and 99.8 percent, respectively. ${ }^{8,9}$ Choi et al ${ }^{10}$ further concluded that polymerase chain reaction is the method of choice for diagnosis of $M$ tuberculosis when it is suspected clinically but smears or cultures are negative. They further describe an added role for polymerase chain reaction in facilitating rapid distinction between $M$ tuberculosis and other mycobacterial infections, when smears are positive.

Rapid assays such as the polymerase chain reaction have great potential for identifying infections involving pathogens such as $M$ tuberculosis that are difficult, slow, or impossible to culture in vitro, especially when delayed diagnosis can be catastrophic. The polymerase chain reaction technique is, however, fraught with many practical challenges, and its routine application in clinical laboratories is impeded by inhibitors in clinical specimens and the possible false-positive reactions caused by contaminating amplicons. ${ }^{4}$ At our laboratory, the assay incorporated several innovations, including internal controls and a sample preparation and amplification protocol designed to increase both sensitivity and specificity.

Optimal management of any brain abscess requires cooperation between physician and neurosurgeon. Successful treatment of pulmonary or extrapulmonary tuberculosis requires the insurance of patient compliance and effective drug regimens of combined pharmacotherapy to prevent the emergence of resistant strains.

Therapy with four drugs (isoniazid, rifampin, ethambutol, and pyrazinamide) for 2 months, followed by two drugs (isoniazid and rifampicin) for 4 months, is advised in most cases of extrapulmonary tuberculosis, particularly for tuberculosis involving the central nervous system, where even a small possibility of isoniazid resistance cannot be risked. First-line drugs for initial therapy include ethambutol and rifampin because they can cross the blood-brain barrier in sufficient bacteriostatic quantities. We followed the American Thoracic Society recommendation of a 2 -month period of daily doses of isoniazid ( $5 \mathrm{mg} / \mathrm{kg}$ up to $300 \mathrm{mg}$ ), rifampin $(10 \mathrm{mg} / \mathrm{kg}$ up to $600 \mathrm{mg})$, and pyrazinamide (20 to $35 \mathrm{mg} / \mathrm{kg}$, up to $2 \mathrm{~g}$ ), and added ethambutol (15 to $25 \mathrm{mg} / \mathrm{kg}$ ). On completion of 2 months of therapy with the above drugs, our patient received an additional 4 months of therapy two to three times per week (directly observed treatment) of isoniazid ( 20 to $40 \mathrm{mg} / \mathrm{kg}$, up to 900 $\mathrm{mg}$ ) and rifampin (10 $\mathrm{mg} / \mathrm{kg}$, up to $600 \mathrm{mg}$ ).

The literature recognizes that early antibiotics alone can cure brain abscesses. ${ }^{11}$ Patients with a lesion on a CT or MRI scan consistent with bacterial abscess can begin empiric therapy even if urgent neurosurgical intervention is indicated. Such nonoperative management is most often considered when the patient is neurologically stable and CT or MRI findings are consistent with cerebritis. ${ }^{12,13}$

That said, almost all patients with brain abscess require surgery for optimal management. The timing and type of surgical procedure, that is, excision or aspiration, remain controversial. Patients considered for excision are more often in satisfactory neurologic condition, whereas aspiration is more often performed in the deteriorating, neurologically compromised patient or for drainage of inaccessible lesions that are associated with high mortality rates. ${ }^{14}$ The procedure decided upon is tailored to patient characteristics and is dependent on the clinical course, size, and location of the abscess, CT scan appearance, and other factors. Neurologic deterioration mandates surgery, usually aspiration. If the patient remains stable, and the abscess is accessible, aspiration (CT guided if possible) is preferred, as a specific bacteriologic diagnosis can narrow the antimicrobial regimen.

If the lesion appears encapsulated by CT scan criteria, antibiotic treatment can be started and aspiration for diagnosis and drainage performed without delay. Continued neurologic deterioration or failure of the abscess to decrease as detected by CT scan are indications for surgery, often excision. Excision is also preferred for posterior fossa lesions and for fungal brain abscesses, for which the efficacy of drug therapy is likely to be limited.

Various authors report a high degree of morbidity following surgical excision of tuberculous abscesses, and many have indicated the persistence of symptoms and signs, such as cerebellar ataxia and cranial nerve deficits, 3 to 6 months postoperatively. Whitener ${ }^{5}$ found that most survivors received a dual approach of surgical excision and pharmacotherapy, as occurred with our patient.

In summary, extrapulmonary tuberculosis is 
most often considered a diagnosis of exclusion commonly occurring in immunocompromised persons. The case described here underscores the importance of considering extrapulmonary tuberculosis in patients who have unexplained brain mass lesions and respond poorly to initial therapy for other more common conditions. The case also points out the emerging role of polymerase chain reaction in the diagnosis of tuberculosis and the treatment of a tubercular abscess. The satisfactory outcome of this case was, in all probability, facilitated by the relief of the space-occupying effects of the abscess by drainage and early use of antituberculosis therapy.

\section{References}

1. Elder NC. Extra pulmonary tuberculosis. A review. Arch Fam Med 1992;1:91-8.

2. Inbasekaran $V$, Natarajan $M$. Tuberculous brain abscess. J Indian Med Assoc 1991;89:207-9.

3. Linet MS, Stewart WF, Celentano DD, Ziegler D, Sprecher M. An epidemiologic study of headache among adolescents and young adults. JAMA 1989; 261:2211-6.

4. Nolte FS, Metchock B, McGowan JE, Edwards A, Okwumabua $O$, Thurmond $C$, et al. Direct detection of Mycobacterium tuberculosis in sputum by polymerase chain reaction and DNA hybridization. $\mathrm{J}$ Clin Microbiol 1993;31:1777-82.

5. Whitener DO. Tuberculous brain abscess. Report of a case and review of the literature. Arch Neurol $1978 ; 35: 148-55$.

6. Dastur HM, Desai AD. A comparative study of brain tuberculomas and gliomas based upon 107 case records of each. Brain 1965;88:375-96.

7. Arseni $C$. Two hundred and one cases of intracranial tuberculomas treated surgically. J Neurol Neurosurg Psychiatry 1958;21:308-11.

8. Daniel TM. New approaches to the rapid diagnosis of tuberculous meningitis. J Infect Dis 1987;155: 599-602.

9. Noordhoek GT, Kaan JA, Mulder S, Wilke H, Kolk $\mathrm{AH}$. Routine application of the polymerase chain reaction for detection of Mycobacterium tuberculosis in clinical samples. J Clin Pathol 1995;48:810-4.

10. Choi YJ, Hu Y, Mahmood A. Clinical significance of a polymerase chain reaction assay for the detection of Mycobacterium tuberculosis. Am J Clin Pathol 1996;105:200-4.

11. Rosenblum ML, Mampalam TJ, Pons VG. Controversies in the management of brain abscesses. Clin Neurosurg 1986;33:603.

12. Rosenblum ML, Hoff JT, Norman D, Edwards MS, Berg BO. Nonoperative treatment of brain abscesses in selected high-risk patients. J Neurosurg 1980;52:217-25.

13. Boom WH, Tuazon CU. Successful treatment of multiple brain abscesses with antibiotics alone. Rev Infect Dis 1985;7:189-99.

14. Carey ME: Brain abscesses. Contemp Neurosurg $1982 ; 3: 1$. 